\title{
Photochemical Reactions of 1,3-Diphenyl-1-propen-2,3-one in Polystyrene Solutions
}

\author{
Bolesław Waligora, Maria NowaKowskA, and Joanna KowAL \\ Department of Physical Chemistry and Electrochemistry, \\ Institute of Chemistry, Jagiellonian University, \\ 30-060 Krakow, Karasia 3, Poland.
}

(Received January 22, 1980)

\begin{abstract}
KEY WORDS 1,3-Diphenyl-1-propen-2,3-one / Polystyrene / Trans $\rightarrow$ cis Photoisomerization / Quantum Efficiency / UV Spectral Analysis /
\end{abstract}

$\alpha, \beta$-Enone compounds were found among the carbonyl products of polystyrene photooxidation. In our previous papers, ${ }^{1-3}$ numerous model impurities have been used for elucidating their possible role in actual polymer degradation. The choice of model compounds was based on the identification of products from polystyrene photooxidation. Each of these products was incorporated into a polystyrene sample, and the photochemical processes in such systems were investigated. In this way, it was possible to determine their individual influence on the polymer photooxidation.

$\alpha, \beta$-Enones should be taken into account when the mechanism of polystyrene deterioration is considered as being able to absorb efficiently the near $U V$ radiation.

1,3-Diphenyl-1-propen-2,3-one (chalcone) was chosen as a simple model compound, representing the $\alpha, \beta$-enone products of polystyrene photooxidation. Chalcone trans $\rightarrow$ cis photoisomerization and thermally initiated cis $\rightarrow$ trans isomerization have been studied by several authors. ${ }^{4-7}$

In previous papers ${ }^{8,9}$ the kinetic courses of transchalcone photochemical reactions in various solvents were studied. The following processes were found to occur: 1) trans $\rightarrow$ cis photoisomerization, 2) intermolecular hydrogen abstraction, and 3) reaction between chalcone and singlet oxygen generated in the system. The relative quantum yields depend on the nature of the solvent. These three photochemical processes lead to the consumption of trans-chalcone.

The aim of this paper is to evaluate the photochemical reactivity of trans-chalcone in the presence of polystyrene in $\mathrm{CH}_{2} \mathrm{Cl}_{2}$ and toluene solutions.

Because of the high extinction coefficient of transchalcone and the relatively low extinction coefficients of photochemical reaction products in the spectral range investigated, UV spectrophotometry made possible the monitoring of the trans-chalcone consumption process.

\section{EXPERIMENTAL}

1,3-Diphenyl-1-propen-2,3-one was prepared and purified for spectral analysis in the organic chemistry laboratory at the Jagiellonian University.

Polystyrene was prepared by the thermal polymerization of styrene at $80^{\circ} \mathrm{C}$ for $450 \mathrm{~h}$. The weightaverage molecular weight of this polymer, determined viscometrically, was 462000 .

Toluene and methyl chloride of special grade for spectroscopy were used as solvents.

Chalcone solutions were irradiated with light at $\lambda>305 \mathrm{~nm}$. An ASH 400 medium pressure $\mathrm{Hg}$ lamp and a phthalate liquid filter ${ }^{10}$ were used. The incident radiation intensity at $313 \mathrm{~nm}$ was $2.3 \times 10^{-9}$ Einstein $\mathrm{s}^{-1} \mathrm{~cm}^{-2}$ as determined by ferrioxalate actinometry. ${ }^{11}$

Photochemical experiments were carried out in cylindrical quartz cells of $0.5 \mathrm{~cm}$ thickness in the presence of air at atmospheric pressure.

Polystyrene films of $2 \times 10^{-3} \mathrm{~cm}$ in thickness, containing chalcone, were prepared as described in a previous paper. $^{2}$ 
The UV absorption spectra of the solutions and polystyrene films were recorded with a Zeiss Specord UV-VIS spectrophotometer.

\section{RESULTS AND DISCUSSION}

The near UV $(\lambda>305 \mathrm{~nm})$ irradiation of chalcone solutions in $\mathrm{CH}_{2} \mathrm{Cl}_{2}$ and toluene with the addition of polystyrene at a concentration of $10^{-4}$ to $10^{-1}$ mol $\mathrm{dm}^{-3}$ results in a decrease in chalcone absorption $\left(\lambda_{\max }^{\mathrm{ch}}=307 \mathrm{~nm}\right)$. These absorption changes occur very rapidly. For the toluene solution $\left(c_{\mathrm{ch}}, 3.5 \times 10^{-5} \mathrm{~mol} \mathrm{dm}{ }^{-3} ; c_{\mathrm{PS}}, 7.8 \times 10^{-3} \mathrm{~mol}\right.$ $\left.\mathrm{dm}^{-3}\right)$, the value of absorption at $\lambda_{\text {max }}^{\mathrm{ch}}$ is halved after $25 \mathrm{~s}$ of irradiation.

During the same time interval, an increase in absorption at $\lambda=262 \mathrm{~nm}$ could be observed, thus indicating the formation of cis-chalcone in the trans $\rightarrow$ cis photoisomerization process. ${ }^{5}$

Lutz and Jordan ${ }^{4}$ observed thermally initiated cis $\rightarrow$ trans isomerization of chalcone in the presence of $\mathrm{H}^{+}$ions in an isooctane solution.

Similar results were obtained for $\mathrm{CH}_{2} \mathrm{Cl}_{2}$ solutions of chalcone containing polystyrene at various concentrations, as shown in Figure 1.

It can be noticed that the heating of a solution results in reincrease in absorption in the range of the trans-chalcone absorption. It may thus be con- cluded that trans $\rightarrow$ cis isomerization is the main photochemical process occuring during the early stages of the near UV irradiation of a chalcone + polystyrene solution.

The experiments carried out for solutions at various polystyrene concentrations indicate that an increase in the polystyrene concentration is followed by a decrease in the rate of chalcone consumption process. In general, the rate of trans-chalcone cosumption is given by the equation,

$$
-\frac{\mathrm{d} c_{\mathrm{ch}}}{\mathrm{d} t}=I_{\mathrm{a}} \varphi_{\mathrm{ISC}} \varphi_{\text {cons }}
$$

where,

$I_{\mathrm{a}}$ is the rate of light-absorption process (Einstein $\mathrm{dm}^{-3} \mathrm{~s}^{-1}$ ),

$\phi_{\text {ISC }}$ is the quantum efficiency of trans-chalcone excited triplet-state formation,

$\varphi_{\text {cons }}$ is the quantum efficiency of trans-chalcone cosumption process.

Dissolving eq 1 leads to,

$$
\varphi=\varphi_{\mathrm{ISC}} \varphi_{\text {cons }}=\frac{\Delta c+\frac{1}{\varepsilon l} \ln \frac{1-\mathrm{e}^{-A_{0}}}{1-\mathrm{e}^{-A_{t}}}}{I_{0} \Delta t}
$$

where,

$\Delta c$ is the change in the trans-chalcone con-

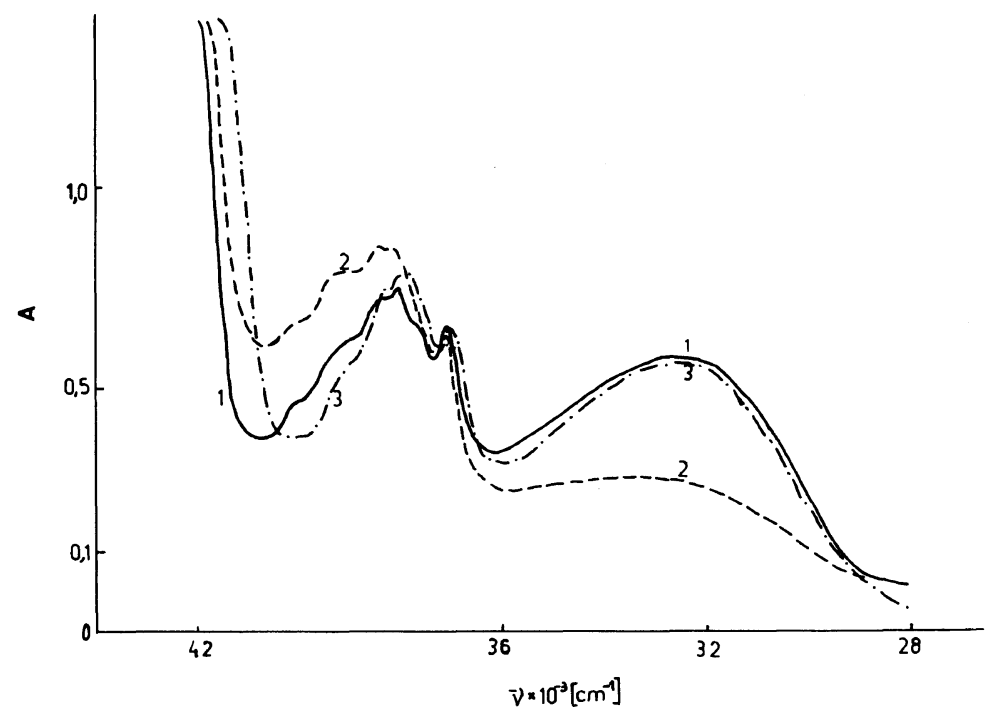

Figure 1. UV absorption spectra of chalcone + polystyrene in $\mathrm{CH}_{2} \mathrm{Cl}_{2}\left(c_{\mathrm{ch}}, 2 \times 10^{-5} \mathrm{~mol} \mathrm{dm}^{-3} ; c_{\mathrm{PS}}\right.$, $2 \times 10^{-3} \mathrm{~mol} \mathrm{dm}^{-3}$ ): 1 , before irradiation; 2, after irradiation with light at $\lambda>305 \mathrm{~nm}$ for $60 \mathrm{~s} ; 3$, after heating at the boiling point of $\mathrm{CH}_{2} \mathrm{Cl}_{2}$ for $5 \mathrm{~min}$ at the presence of $\mathrm{HCl}$. 
centration corresponding to the irradiation time $\Delta t$,

$\varepsilon$ is the molar extinction coefficient at $\lambda_{\max }^{\mathrm{ch}}=307 \mathrm{~nm}$,

$l$ is the thickness of solution layer,

$A_{0}$ is the initial sample absorbance at $\lambda_{\max }^{\mathrm{ch}}=307 \mathrm{~nm}$,

$A_{\mathrm{t}}$ is the sample absorbance after irradiation time $\Delta t$,

$I_{0}$ is the incident light intensity (Einstein $\left.\mathrm{dm}^{-3} \mathrm{~s}^{-1}\right)$.

Figure 2 shows the inter-dependence of the ratio $\varphi_{0} / \varphi$ and polystyrene concentration, where $\varphi_{0}$ and $\varphi$ are the quantum efficiencies of the trans-chalcone consumption process in a toluene solution with and without the presence of polystyrene, respectively.

The experimental data presented in Figure 2 show that the quantum efficiency of the transchalcone consumption process decreases as the polystyrene concentration increases.

Trans $\rightarrow$ cis chalcone photoisomerization could also be observed when trans-chalcone was incorporated into the polystyrene film but the rate and quantum efficiency of this process were considerably reduced $\left(\varphi_{0} / \varphi \approx 10^{2}\right)$, owing perhaps to the increase in the viscosity of the system.

The results obtained here indicate that in the initial period of the irradiation of PS + chalcone samples (solutions, films), the photooxidation of polystyrene does not occur because of trans $\rightarrow$ cis photoisomerization of chalcone, which is the predominant process in the system. It was noticed, however, that the photoisomerization was followed by much slower secondary processes in which chalcone was also consumed. The sensitized photooxidation of polystyrene films connected with the second step of the chalcone consumption process is the subject of present investigation.

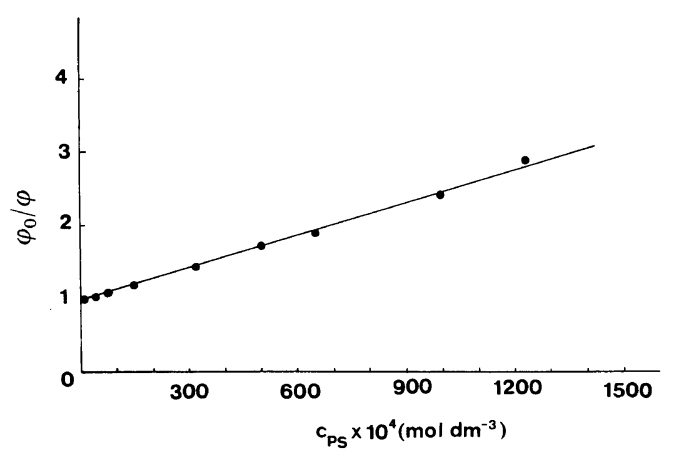

Figure 2. Dependence of $\varphi_{0} / \varphi\left(\varphi_{0}\right.$, quantum efficiency of the trans-chalcone consumption process in the absence of polystyrene; $\varphi$, quantum efficiency of trans-chalcone consumption in the presence of polystyrene) on the polystyrene concentration.

\section{REFERENCES}

1. J. Kubica, Makromol. Chem., 178, 3017 (1977).

2. J. Kubica, Eur. Polym. J., 13, 325 (1977).

3. J. Kowal and M. Nowakowska, Polymer, 20, 1003 (1979); Polymer, in press.

4. R. E. Lutz and R. H. Jordan, J. Am. Chem. Soc., 72, 4090 (1950).

5. D. S. Noyce, W. A. Pryor, and P. A. King, J. Am. Chem. Soc., 81, 5423 (1959).

6. W. A. Black and R. E. Lutz, J. Am. Chem. Soc., 77, 5134 (1959).

7. D. S. Noyce and M. J. Jorgenson, J. Am. Chem. Soc., 83, 2525 (1961).

8. M. Nowakowska and J. Kowal, Bull. Pol. Acad. Polon. Sci., Ser. Sci. Chim., 27, 409 (1979).

9. J. Kowal and M. Nowakowska, Bull. Pol. Acad. Polon. Sci., Ser. Sci. Chim., in press.

10. J. G. Calvert and J. N. Pitts, Jr., "Photochemistry," Wiley, New York, N.Y., 1966.

11. G. A. Parker, "Photoluminescence of Solutions," Elsevier, Amsterdam, 1968. 